\title{
Synthesis of novel bis-allyloxy and hydroxypropoxy derivatives of 4, 5-diaryl thiophene-2-carboxylic acid and their biological evaluation
}

\author{
T SHANMUGANATHAN ${ }^{\mathrm{a}, \mathrm{b}, *}, \mathrm{M} \mathrm{VENUGOPAL}^{\mathrm{c}}, \mathrm{K}$ PARTHASARATHY ${ }^{\mathrm{d}}$, \\ N DHATCHANAMOORTHY ${ }^{\mathrm{a}}$, Y ARUN ${ }^{\mathrm{e}}$ and A A M PRINCE ${ }^{\mathrm{b}}$ \\ ${ }^{a}$ Orchid Pharma Ltd, R\&D Centre, Chennai, Tamilnadu 600 119, India \\ ${ }^{b}$ Department of chemistry, Ramakrishna Mission Vivekananda College, Mylapore, Chennai, Tamilnadu \\ 600 004, India \\ ${ }^{c}$ Ven Biotech Private Limited, Chennai, Tamilnadu 600 095, India \\ ${ }^{\mathrm{d}}$ Department of Chemistry, Siddha Central Research Institute, Central Council for Research in Siddha, Chennai, \\ Tamilnadu 600 106, India \\ ${ }^{\text {e}}$ Organic Chemistry Division, Central Leather Research Institute (CSIR), Adyar, Chennai, Tamilnadu \\ 600 020, India \\ Email: t_shanmuganathan09@yahoo.com
}

MS received 5 January 2017; revised 1 April 2017; accepted 2 April 2017

\begin{abstract}
In our earlier studies, we have shown that the introduction of amino moieties at carboxylic acid of 4,5-diarylthiophene-2-carboxylic acid significantly improved the anti-inflammatory activity of the compound against the standard drug diclofenac sodium. In the present study, we have synthesized new derivatives of 4,5diarylthiophene-2-carboxylic acid by modifying the hydroxyl group of the phenyl ring and carboxylic acid group of the thiophene ring. A series of novel 4,5-diarylthiophene-2-carboxylic acid derivatives containing bis-allyloxy and hydroxypropoxy with methyl or ethyl ester moieties were synthesized, characterized and subsequently evaluated for anti-inflammatory and antioxidant property. Among the novel compounds, the inhibition of bovine serum albumin denaturation assay revealed that the compound 4,5-bis(4-(3-hydroxypropoxy)phenyl)thiophene2-carboxylic acid (15) and ethyl ester (13) having anti-inflammatory activity better than the standard drug diclofenac sodium. The antioxidant screening showing 4,5-bis(4-(allyloxy)phenyl)thiophene-2-carboxylic acid (10), 4,5-bis(4-(3-hydroxypropoxy)phenyl)thiophene-2-carboxylic acid methyl ester (11) and 4,5-bis(4-(3hydroxypropoxy)phenyl)thiophene-2-carboxylic acid ethyl ester (13) exhibited a slightly moderate antioxidant activity than standard ascorbic acid. Molecular docking analysis was performed for the synthesized compounds with the cyclooxygenase-2 (COX-2) receptor (PDB 1D: 1PXX). Docking studies revealed that all the synthesised compounds exhibit greater binding affinity than the standard drug. Particularly, the compound ethyl 4,5-bis(4(allyloxy)phenyl)thiophene-2-carboxylate (8) and allyl 4,5-bis(4-(allyloxy)phenyl)thiophene-2-carboxylate (9) having high free energy binding of -10.40 and $-10.48 \mathrm{Kcal} / \mathrm{mol}$, respectively.
\end{abstract}

Keywords. Bis-allyloxy derivatives; hydroxypropoxy derivatives; 4,5-diarylthiophene-2-carboxylic acid; anti-inflammatory; antioxidant; molecular docking.

\section{Introduction}

Non-steroidal anti-inflammatory drugs (NSAIDs) are therapeutic agents commonly used in the treatment of inflammation, pain and fever. The therapeutic effect of these substances involves inhibition of cyclooxygenases (COX) thereby preventing prostaglandin (PGs) and thromboxane $\mathrm{A} 2$ formation from arachidonic acid. ${ }^{1}$ There are two isoforms, COX-1 and COX-2, where inhibition of COX-1 is responsible, in part, for gastrointestinal and renal side effects, whereas selective COX-2

\footnotetext{
*For correspondence
}

inhibitors, have been designed, synthesized and clinically introduced as gastrointestinal sparing NSAIDs. Hence, the beneficial effect of selective inhibition of COX-2 over COX-1 has triggered therapeutic interest on the former, resulting in the evolution of drugs with reduced side effect to the patients.

The general structure of COX-2 selective inhibitors consist of two aryl groups attached to neighboring atoms of a central ring, one of the aryl groups is parasubstituted with either a methyl sulfonyl $\left(\mathrm{SO}_{2} \mathrm{CH}_{3}\right)$ group, e.g., etoricoxib and rofecoxib or a sulfonamide $\left(\mathrm{SO}_{2} \mathrm{NH}_{2}\right)$, e.g., celecoxib and parecoxib. ${ }^{2}$ The central 
<smiles>O=C(O)Cc1ccccc1Nc1c(F)cccc1Cl</smiles>

Lumiracoxib<smiles>CC(C(=O)O)c1ccc(Oc2ccccc2)cc1</smiles>

Fenoprofen<smiles>O=C(O)Cc1ccccc1Nc1c(Cl)cccc1Cl</smiles>

Diclofenac<smiles>COc1ccc2c(c1)c(CC(=O)O)c(C)n2C(=O)c1ccc(Cl)cc1</smiles>

Indomethacin<smiles>C=CCOc1ccc(CC(=O)O)cc1Cl</smiles>

Alclofenac

Flurbiprofen<smiles>CC(C)Cc1ccc(C(C)C(=O)O)cc1</smiles>

Ibuprufen

Figure 1. Some of the non-steroidal anti-inflammatory drugs.

rings of compounds are thiophene, pyrazole, furanone, isoxazole, and cyclopentene. There are also examples of cycloalkyl, alkoxy or phenoxy moieties in the non-sulfonyl containing 'aryl' ring. ${ }^{3-5}$ One of the important heterocyclic systems which attract progressive interest of many researchers is thiophene, because of plethora of biological, pharmacological and industrial importance. Thiophene containing drugs exhibit a variety of biological activities which include antimicrobials, antipsychotics, ${ }^{6}$ sedatives, ${ }^{7-9}$ antidepressants (e.g., Duloxetine and Trazodone) and antihypertensive (e.g., Tiamenidine) drugs. Thiophene derivatives are also well known for their pronounced anti-inflammatory activities as evident from the approval of Lornoxicam, Tenoxicam, Tiaprofenic acid and Tenidap by the US Food and Drug Administration (FDA). Recently, several new Thiophene-2-carboxylic acid derivatives have been reported as potent HCV NS5B polymerase inhibitors, ${ }^{10}$ 5-LOX/COX-inhibitor, ${ }^{11}$ and with anti-inflammatory activities. ${ }^{12}$ The introduction of carboxylic acid into a biologically active compound not only impacts the water solubility of the compound but also establishes relatively strong electrostatic interactions or hydrogen-bond bridges with the COX enzymes, conferring both binding affinity and specificity to the drug-target as in the case of flurbiprofen. ${ }^{13}$ Also, carboxylic acid moiety is critical for their biological activity of several NSAID's like aspirin, ibuprofen, naproxen, indomethacin, diclofenac, and lumiracoxib (Figure 1). In addition, esters and amides of indomethacin indeed are more COX2 selective and possess potent anti-inflammatory effects without being ulcerogenic. ${ }^{14}$ Another analogue of diclofenac with a carboxylic moiety and a selective COX2 inhibitor lumiracoxib was reported with its carboxylate group forming hydrogen bonding interactions with Ser530 and Tyr385 at the top of the active site. ${ }^{15}$ These findings have encouraged us to replace the amide moiety with carboxylic acid group of the previously reported potent 4,5diarylthiophene-2-carboxamide derivatives. ${ }^{16}$ In view of these observations and in continuance of our effort to develop novel thiophene analogues, ${ }^{16}$ it was thought worthwhile to synthesize new allyloxy and hydroxypropoxy derivatives of 4,5-diarylthiophene-2-carboxylic acid and to evaluate them for their anti-inflammatory and antioxidant properties.

\section{Experimental}

\subsection{Materials and methods}

All the chemicals and reagents used were lab grade material procured from Alfa aesar, India. All the solvents used were purchased from commercial suppliers and were used without further purification. The melting points were determined using Buchi apparatus by the open capillary tube method and were uncorrected. The IR spectra were recorded in PerkinElmer series 2000 FTIR spectrophotometer using KBr pellet. ${ }^{1} \mathrm{H}$ NMR and ${ }^{13} \mathrm{C}$ NMR spectra were obtained in $\mathrm{CDCl} 3$, DMSO- $d_{6}$ on a Bruker spectrometer at 400 and $100 \mathrm{MHz}$, respectively. The chemical shifts are reported in ppm $(\delta)$ relative to tetramethylsilane as internal standard, coupling constants $(J)$ are in hertz $(\mathrm{Hz})$. Spin multiplicities are given as s (singlet), d (doublet), t (triplet), dd (doublet of doublet), bs (broad signal) and $\mathrm{m}$ (multiplet). Residual proton and carbon solvent signal for $\mathrm{CDCl} 3, \delta_{\mathrm{H}} 7.26 \mathrm{ppm}, \delta_{\mathrm{C}} 77.0 \mathrm{ppm}, \delta_{6}$ DMSO, $\delta_{\mathrm{H}} 2.50 \mathrm{ppm}, \delta_{\mathrm{C}} 40.0 \mathrm{ppm}$. Proton and carbon spectra 
were typically obtained at room temperature. Mass spectra were recorded on ESI-Perkin Elmer Sciex, API 3000 mass spectrometer. Pre-coated silica gel GF254 plates from Merck were used for thin layer chromatography (TLC). The elemental analyses were recorded in Thermo Finnigan Flash EA 1112 elemental analyser.

\subsection{Synthesis}

2.2a Synthesis of 2,3-bis(4-methoxy phenyl)-3-chloro2-prop-2-ene aldehyde (2): Phosphorous oxychloride $\left(\mathrm{POCl}_{3}\right)$ (7.66 g, 0.05 mole) was added drop wise over a period of $15-30 \mathrm{~min}$ with stirring at $0-5^{\circ} \mathrm{C}$ to $20 \mathrm{~mL}$ of dimethylformamide. The mass was maintained at $0-5^{\circ} \mathrm{C}$ over $30 \mathrm{~min}$ and a solution of desoxyanisoin (10 g, 0.04 mole) in dimethylformamide $(50 \mathrm{~mL})$ at $0-5^{\circ} \mathrm{C}$ was added under stirring. The reaction mass was heated to $70-75^{\circ} \mathrm{C}$ and maintained for $4 \mathrm{~h}$. The progress of the reaction was monitored using TLC (toluene). After completion, the reaction mixture was cooled and poured slowly into $25 \%$ solution of sodium acetate in water $(100 \mathrm{~mL})$. The product was filtered and washed with water, followed by slurry wash with ethanol (100 mL) which after drying gave $9.1 \mathrm{~g} \mathrm{(78 \%} \mathrm{yield)} \mathrm{of} \mathrm{the}$ title compound 2 . White powder, M.p.: $145-147^{\circ} \mathrm{C}$. [Lit. $^{12}$; $\left.158^{\circ} \mathrm{C}\right]$. IR(KBr) cm ${ }^{-1}: 2932,1680,1513,1442,1080,813$, 772. ${ }^{1} \mathrm{H} \mathrm{NMR}\left(400 \mathrm{MHz}, \mathrm{CDCl}_{3}\right): \delta 9.66(\mathrm{~s}, 1 \mathrm{H}), 7.52$ $(\mathrm{d}, J=7.8 \mathrm{~Hz}, 2 \mathrm{H}), 7.25-7.22(\mathrm{~m}, 2 \mathrm{H}), 6.99-6.91(\mathrm{~m}, 4 \mathrm{H})$, 3.89 (s, 3H), 3.85 (s, 3H). ${ }^{13} \mathrm{C}$ NMR (100 MHz, CDCl3): $\delta$ 190.5, 162.1, 159.7, 155.1, 139.8, 132.4, 132.1, 131.1, 130.6, 128.4, 126.7, 114.2, 113.9, 55.8, 55.5. ESI-MS m/z Calculated 302.1. Found: $303.1[\mathrm{M}+\mathrm{H}]^{+}$.

2.2b Synthesis of 4,5-bis(4-methoxyphenyl)thiophene2-carboxylic acid (3): 2,3-Bis(4-methoxy phenyl)-3chloro-2-prop-2-ene aldehyde 2 ( $5 \mathrm{~g}, 0.0165 \mathrm{~mol}$ ) was added to a solution of potassium hydroxide $(4 \mathrm{~g}, 0.714 \mathrm{~mol})$ and 2-mercapto acetic acid (3.1 g, $0.034 \mathrm{~mol})$ in methanol: water $(40 \mathrm{~mL}: 10 \mathrm{~mL})$ mixture at room temperature. The mixture was refluxed for $4 \mathrm{~h}$, and reaction was monitored using TLC (hexane: ethyl acetate 3:7). After completion, the reaction mixture was cooled to room temperature and slowly acidified with concentrated $\mathrm{HCl}$ over $30-45 \mathrm{~min}$ at $25-30^{\circ} \mathrm{C}$. The product 3 was filtered, washed with water and dried to get compound 3. $4 \mathrm{~g}$ (71\% yield). Yellow powder, M.p.: $211-213^{\circ} \mathrm{C}$. [Lit. ${ }^{12}$; $\left.215^{\circ} \mathrm{C}\right]$. IR (KBr) cm $\mathrm{cm}^{-1}: 2933,2542,1668,1546,1449,1247$, 1033, 827. ${ }^{1} \mathrm{H}$ NMR $\left(400 \mathrm{MHz}, \mathrm{CDCl}_{3}\right): \delta 10.7(\mathrm{bs}, 1 \mathrm{H}), 7.87$ (s, $1 \mathrm{H}), 7.27(\mathrm{~d}, J=8.6 \mathrm{~Hz}, 2 \mathrm{H}), 7.20(\mathrm{~d}, J=8.6 \mathrm{~Hz}, 2 \mathrm{H})$, 6.85-6.81 (m, 4H), 3.83 (s, 3H), $3.81(\mathrm{~s}, 3 \mathrm{H}) .{ }^{13} \mathrm{C}$ NMR $\left(100 \mathrm{MHz}, \mathrm{CDCl}_{3}\right): \delta 168.0,159.9,159.1,147.0,138.4$, 137.9, 130.6, 130.3, 129.5 128.1, 125.9, 114.3, 114.1, 55.5, 55.4. ESI-MS $\mathrm{m} / z$ Calculated 340.1. Found: $339.1[\mathrm{M}-\mathrm{H}]^{-}$.

2.2c Synthesis of 4,5-bis(4-hydroxyphenyl)thiophene2-carboxylic acid (4): 4,5-Bis(4-methoxyphenyl) thiophene-2-carboxylic acid 3 (6 g, $0.0176 \mathrm{~mol})$ was added to the mixture of aluminium chloride $(9.38 \mathrm{~g}, 0.0704 \mathrm{~mol})$ in chlorobenzene $(60 \mathrm{~mL})$ at $25-30^{\circ} \mathrm{C}$. The reaction mixture was heated to $95-105^{\circ} \mathrm{C}$ and maintained for $3 \mathrm{~h}$. The progress of the reaction was monitored using TLC (hexane:ethyl acetate 3:7). After completion of the reaction, aqueous $\mathrm{HCl}(1: 1,50 \mathrm{~mL})$ was added to the mass at $25-30^{\circ} \mathrm{C}$. The product was extracted with ethyl acetate $(50 \mathrm{~mL} \times 2)$ and the organic layer was washed with water. Ethyl acetate was evaporated under vacuum and the residue was triturated with dichloromethane $(100 \mathrm{~mL})$, filtered to get compound 4 . $4.85 \mathrm{~g}$ (88\% yield). Light green powder, M.p.: $240-243^{\circ} \mathrm{C}$. $\operatorname{IR}(\mathrm{KBr}) \mathrm{cm}^{-1}: 3428,3318,1639,1545,1442,1255,1069$, 829. ${ }^{1} \mathrm{H}$ NMR $\left(400 \mathrm{MHz}, \mathrm{CDCl}_{3}+\mathrm{DMSO}-d_{6}\right): \delta 8.48$ (bs, 1H), 8.19 (bs, 1H), 7.75 (s, 1H), 7.17 (d, $J=8.5 \mathrm{~Hz}, 2 \mathrm{H})$, $7.11(\mathrm{~d}, J=8.4 \mathrm{~Hz}, 2 \mathrm{H}), 6.77-6.75(\mathrm{~m}, 4 \mathrm{H}) .{ }^{13} \mathrm{C} \mathrm{NMR}$ $\left(100 \mathrm{MHz}, \mathrm{CDCl}_{3}+\mathrm{DMSO}-d_{6}\right): \delta 164.3,157.5,156.4,144.9$, $137.8,136.2,130.9,130.4,130.0,127.1,124.9,115.6,115.5$. ESI-MS $m / z$ Calculated 312.0. Found: $313.0[\mathrm{M}+\mathrm{H}]^{+}$.

2.2d Synthesis of methyl 4,5-bis(4-hydroxyphenyl) thiophene-2-carboxylate (5): 4,5-Bis(4-hydroxyphenyl) thiophene-2-carboxylic acid (3.12 g, $0.01 \mathrm{~mol})$ dissolved in methanol $(78 \mathrm{~mL})$ and concentrated sulphuric acid $(1.0 \mathrm{~g}$, $0.01 \mathrm{~mol}$ ) was added slowly and refluxed for $8 \mathrm{~h}$. The reaction was monitored by using TLC (hexane: ethyl acetate 3:7). After the reaction was completed, the reaction mixture was cooled and concentrated. To this residue $20 \mathrm{~mL}$ of water added, the product was extracted with ethyl acetate $(2 \times 50 \mathrm{~mL})$. The organic phase was separated, washed with water, $10 \%$ sodium carbonate solution followed by water and evaporated under vacuum. The residue was triturated with heptane $(20 \mathrm{~mL})$, filtered to get compound 5. $2.8 \mathrm{~g}$ ( $86 \%$ yield). The product obtained did not require any further purification for next step.

Off-white powder, M.p.: $211-213^{\circ} \mathrm{C}$. IR $(\mathrm{KBr}) \mathrm{cm}^{-1}$ : $3325,2949,1664,1547,1441,1227,1079,834,756 .{ }^{1} \mathrm{H}$ NMR $\left(400 \mathrm{MHz}, \mathrm{CDCl}_{3}+\mathrm{DMSO}_{6}\right) \delta 8.12(\mathrm{~s}, 1 \mathrm{H}), 7.76(\mathrm{~s}$, $2 \mathrm{H}), 7.17(\mathrm{~d}, 2 \mathrm{H}, J=8.44 \mathrm{~Hz}), 7.10(\mathrm{~d}, 2 \mathrm{H}, J=8.4 \mathrm{~Hz})$, 6.77-6.75 (m, 4H), 3.89 (s, 3H). ${ }^{13} \mathrm{C}$ NMR $(100 \mathrm{MHz}$, $\left.\mathrm{CDCl}_{3}+\mathrm{DMSO}_{-} \mathrm{d}_{6}\right) \delta 162.9,157.5,156.4,145.4,137.9$, $136.5,130.4,130.1,129.5,127.0,124.8,115.7,115.6,52.12$. ESI-MS $m / z$ Calculated 326.1. Found: $327.1[\mathrm{M}+\mathrm{H}]^{+}$. Anal. Calculated for $\mathrm{C}_{18} \mathrm{H}_{14} \mathrm{O}_{4} \mathrm{~S}: \mathrm{C}, 66.24 ; \mathrm{H}, 4.32 \%$. Found: $\mathrm{C}$, $66.28 ; \mathrm{H}, 4.30 \%$.

\section{2e Synthesis of ethyl 4,5-bis(4-hydroxyphenyl)} thiophene-2-carboxylate (6): 4,5-Bis(4-hydroxyphenyl) thiophene-2-carboxylic acid $(3.12 \mathrm{~g}, 0.01 \mathrm{~mol})$ dissolved in ethanol $(78 \mathrm{~mL})$ and concentrated sulphuric acid $(1.0 \mathrm{~g}$, $0.01 \mathrm{~mol}$ ) was added slowly and refluxed for $8 \mathrm{~h}$. The reaction was monitored by using TLC (hexane:ethyl acetate 3:7). After the reaction was completed, the reaction mixture was cooled and concentrated. To this residue water $(20 \mathrm{~mL})$ was added and the product was extracted with ethyl acetate $(2 \times 50 \mathrm{~mL})$. The organic phase was separated, washed with water, $10 \%$ sodium carbonate solution followed by water and evaporated under vacuum. The residue was triturated with heptane (20 mL), filtered to get compound 6. $3.0 \mathrm{~g}$ (88\% yield). The 
product obtained did not require any further purification for next step.

Yellow powder, M.p.: $202-204^{\circ} \mathrm{C}$. IR $(\mathrm{KBr}) \mathrm{cm}^{-1}$ : 3317 , 2937, 1656, 1547, 1442, 1228, 1077, 833, 756. ${ }^{1} \mathrm{H}$ NMR $\left(400 \mathrm{MHz}, \mathrm{CDCl}_{3}+\mathrm{DMSO}_{-} \mathrm{d}_{6}\right) \delta 9.19(\mathrm{~s}, 1 \mathrm{H}), 8.99(\mathrm{~s}, 1 \mathrm{H})$, $7.71(\mathrm{~s}, 1 \mathrm{H}), 7.14(\mathrm{~d}, 2 \mathrm{H}, J=8.56 \mathrm{~Hz}), 7.08(\mathrm{~d}, 2 \mathrm{H}, J=$ $8.52 \mathrm{~Hz}), 6.76-6.73(\mathrm{~m}, 4 \mathrm{H}), 4.37(\mathrm{q}, 2 \mathrm{H}, J=7.08 \mathrm{~Hz})$, 1.39 (t, $3 \mathrm{H}, J=7.12 \mathrm{~Hz}) .{ }^{13} \mathrm{C} \mathrm{NMR}\left(100 \mathrm{MHz}, \mathrm{CDCl}_{3}+\right.$ DMSO-d $\left.\mathrm{d}_{6}\right) \delta 160.9,156.8,155.7,143.8,136.8,134.9,129.2$, 128.9, 125.4, 123.4, 123.2, 114.6, 114.5, 59.86, 13.36. ESIMS $m / z$ Calculated 340.1. Found: $341.1[\mathrm{M}+\mathrm{H}]^{+}$. Anal. Calculated for $\mathrm{C}_{19} \mathrm{H}_{16} \mathrm{O}_{4} \mathrm{~S}: \mathrm{C}, 67.04 ; \mathrm{H}, 4.74 \%$. Found: $\mathrm{C}$, $67.08 ; \mathrm{H}, 4.72 \%$.

\section{$2.2 \mathrm{f}$ Synthesis of methyl 4,5-bis(4-(allyloxy)phenyl)} thiophene-2-carboxylate (7): Allyl bromide (2.5 g, $0.02 \mathrm{~mol}$ ) was added to the mixture of methyl 4,5-bis(4hydroxyphenyl)thiophene-2-carboxylate $(2.5 \mathrm{~g}, 0.008 \mathrm{~mol})$, potassium carbonate $(2.77 \mathrm{~g}, 0.02 \mathrm{~mol})$ in acetonitrile $(50 \mathrm{~mL})$. The reaction mixture was refluxed for $4 \mathrm{~h}$. The reaction was monitored by using TLC (hexane: ethyl acetate 1:1). After the completion of the reaction, the reaction mixture was cooled and filtered to remove insolubles. The organic mass was evaporated under vacuum and to this residue water $(20 \mathrm{~mL})$ was added and the product was extracted with ethyl acetate $(2 \times 50 \mathrm{~mL})$. The organic phase was separated, washed with water, and evaporated under vacuum. The residue was triturated with hexane $(20 \mathrm{~mL})$, cooled to $10-15^{\circ} \mathrm{C}$ and filtered to get compound 7. $2.7 \mathrm{~g}$ (87\% yield). The product obtained was used for next step without further purification. Pale brown powder, M.p.: $66-68^{\circ} \mathrm{C}$. IR $(\mathrm{KBr}) \mathrm{cm}^{-1}: 3390,2946,2862$, 1704, 1651, 1543, 1440, 1242, 1073, 833, 754. ${ }^{1} \mathrm{H}$ NMR $\left(400 \mathrm{Mz}, \mathrm{CDCl}_{3}\right) \delta 7.78(\mathrm{~s}, 1 \mathrm{H}), 7.24(\mathrm{~d}, 2 \mathrm{H}, J=6.76 \mathrm{~Hz})$, $7.18(\mathrm{~d}, 2 \mathrm{H}, \mathrm{J}=6.76 \mathrm{~Hz}), 6.85-6.82(\mathrm{~m}, 4 \mathrm{H}), 6.07-6.02$ $(\mathrm{m}, 2 \mathrm{H}), 5.44-5.39(\mathrm{~m}, 2 \mathrm{H}), 5.31-5.28(\mathrm{~m}, 2 \mathrm{H}), 4.54-4.52$ $(\mathrm{m}, 4 \mathrm{H}), 3.89$ (s, 3H). ${ }^{13} \mathrm{C}$ NMR $\left(100 \mathrm{MHz}, \mathrm{CDCl}_{3}\right) \delta 162.9$, $158.8,157.9,145.0,137.9,136.4,133.3,133.1,130.5,130.3$, $130.2,128.3,126.1,118.0,117.87,114.9,114.8,68.9,68.8$, 52.25. ESI-MS m/z Calculated 406.1. Found: $407.1[\mathrm{M}+\mathrm{H}]^{+}$. Anal. Calculated for $\mathrm{C}_{24} \mathrm{H}_{22} \mathrm{O}_{4} \mathrm{~S}: \mathrm{C}, 70.91 ; \mathrm{H}, 5.46 \%$. Found: C, $70.95 ; \mathrm{H}, 5.48 \%$.

2.2g Synthesis of ethyl 4,5-bis(4-(allyloxy)phenyl) thiophene-2-carboxylate (8): Allyl bromide (2.5 g $0.02 \mathrm{~mol})$ was added to the mixture of ethyl 4,5-bis(4hydroxyphenyl)thiophene-2-carboxylate $(2.7 \mathrm{~g}, 0.008 \mathrm{~mol})$, potassium carbonate $(2.77 \mathrm{~g}, 0.02 \mathrm{~mol})$ in acetonitrile $(50 \mathrm{~mL})$. The reaction mixture was refluxed for $4 \mathrm{~h}$. The reaction was monitored by using TLC (hexane:ethyl acetate 1:1). After the reaction was completed, the reaction mixture was cooled and filtered to remove insolubles. The organic mass was evaporated under vacuum and to this residue water $(20 \mathrm{~mL})$ was added and the product was extracted with ethyl acetate $(2 \times 50 \mathrm{~mL})$. The organic phase was separated, washed with water, and evaporated under vacuum. The residue was triturated with hexane $(20 \mathrm{~mL})$, cooled to $10-15^{\circ} \mathrm{C}$ filtered to get compound 8. $2.94 \mathrm{~g}$ (88\% yield). The product obtained was used for next step without further purification.

Yellow powder, M.p.: $65-67^{\circ} \mathrm{C}$ IR $(\mathrm{KBr}) \mathrm{cm}^{-1}: 3429$, 2925, 2862, 1696, 1650, 1546, 1441, 1247, 1073, 827, 755. ${ }^{1} \mathrm{H}$ NMR $\left(400 \mathrm{MHz}, \mathrm{CDCl}_{3}\right) \delta 7.78(\mathrm{~s}, 1 \mathrm{H}), 7.24$ $(\mathrm{d}, 2 \mathrm{H}, J=8.76 \mathrm{~Hz}), 7.18(\mathrm{~d}, 2 \mathrm{H}, \mathrm{J}=8.68 \mathrm{~Hz}), 6.85-6.82$ $(\mathrm{m}, 4 \mathrm{H}), 6.11-6.00(\mathrm{~m}, 2 \mathrm{H}), 5.44-5.39(\mathrm{~m}, 2 \mathrm{H}), 5.31-$ $5.28(\mathrm{~m}, 2 \mathrm{H}), 4.54-4.52(\mathrm{~d}, 4 \mathrm{H}, J=5.32 \mathrm{~Hz}), 4.39-4.33$ $(\mathrm{q}, 2 \mathrm{H}, J=7.12 \mathrm{~Hz}), 1.40(\mathrm{t}, 3 \mathrm{H}, J=7.12 \mathrm{~Hz}) \cdot{ }^{13} \mathrm{C}$ NMR $\left(100 \mathrm{MHz}, \mathrm{CDCl}_{3}\right) \delta 162.4,158.7,157.9,144.8,137.8$, 136.1, 133.2, 133.1, 133.0, 132.9, 130.7, 130.4, 130.1, 128.3, 126.1, 117.9, 117.8, 114.8, 114.7, 114.7, 114.6, 68.8, 68.7, 61.2, 14.4. ESI-MS $\mathrm{m} / \mathrm{z}$ Calculated 420.1. Found: 421.3 $[\mathrm{M}+\mathrm{H}]^{+}$. Anal. Calculated for $\mathrm{C}_{25} \mathrm{H}_{24} \mathrm{O}_{4} \mathrm{~S}: \mathrm{C}, 71.40 ; \mathrm{H}$, 5.75\%. Found: C, 71.44; H, 5.78\%.

2.2h Synthesis of allyl 4,5-bis(4-(allyloxy)phenyl) thiophene-2-carboxylate (9): Allyl bromide (4.2 g, $0.035 \mathrm{~mol}$ ) was added to the mixture of ethyl 4,5-bis(4hydroxyphenyl)thiophene-2-carboxylic acid (3 g, $0.01 \mathrm{~mol}$ ), potassium carbonate $(4.8 \mathrm{~g}, 0.035 \mathrm{~mol})$ in acetonitrile $(75 \mathrm{~mL})$. The reaction mixture was refluxed for $4 \mathrm{~h}$. The reaction was monitored by using TLC (hexane:ethyl acetate 1:1). After the reaction was completed, the reaction mixture was cooled and filtered to remove insolubles. The organic mass was evaporated under vacuum and to this residue water $(20 \mathrm{~mL})$ was added and the product was extracted with ethyl acetate $(2 \times 50 \mathrm{~mL})$. The organic phase was separated, washed with water, and evaporated under vacuum to get compound 9 . $3.4 \mathrm{~g}$ (82\% yield). Brown liquid, IR (KBr) cm $\mathrm{cm}^{-1}: 2924,2862$, 1709, 1547, 1443, 1243, 1069, 831, 753. ${ }^{1} \mathrm{H}$ NMR $(400 \mathrm{MHz}$, $\left.\mathrm{CDCl}_{3}\right) \delta 7.72(\mathrm{~s}, 1 \mathrm{H}), 7.16(\mathrm{~d}, 2 \mathrm{H}, J=8.4 \mathrm{~Hz}), 7.10(\mathrm{~d}, 2 \mathrm{H}$, $J=8.4 \mathrm{~Hz}), 6.77-6.74(\mathrm{~m}, 4 \mathrm{H}), 6.01-5.91(\mathrm{~m}, 3 \mathrm{H}), 5.36-$ $5.31(\mathrm{~m}, 3 \mathrm{H}), 5.22-5.20(\mathrm{~m}, 3 \mathrm{H}), 4.73(\mathrm{~d}, 2 \mathrm{H}, J=5.32 \mathrm{~Hz})$, $4.45(\mathrm{~d}, 4 \mathrm{H}, J=5 \mathrm{~Hz}) .{ }^{13} \mathrm{C}$ NMR $\left(100 \mathrm{MHz}, \mathrm{CDCl}_{3}\right) \delta$ 201.7, 162.1, 158.9, 158.0, 145.3, 138.0, 136.5, 133.3, 133.1, 132.2, 130.6, 130.4, 130.2, 128.4, 126.2, 118.5, 118.0, 117.9, 114.9, 114.8, 68.9, 65.7, 31.1. ESI-MS $m / z$ Calculated 432.1. Found: $433.2[\mathrm{M}+\mathrm{H}]^{+}$. Anal. Calculated for $\mathrm{C}_{26} \mathrm{H}_{24} \mathrm{O}_{4} \mathrm{~S}$ : C, 72.20; H, 5.59\%. Found: C, 72.26; H, 5.56\%.

\section{$2.2 \mathrm{i} \quad$ 4,5-Bis(4-(allyloxy)phenyl)thiophene-2-carboxylic} acid (10): Ethyl 4,5-bis(4-(allyloxy)phenyl)thiophene-2carboxylate $(0.6 \mathrm{~g}, 0.0014 \mathrm{~mol})$ was added at room temperature to a solution of sodium hydroxide $(0.54 \mathrm{~g}, 0.0135 \mathrm{~mol})$ in ethanol:water $(8 \mathrm{~mL}: 2 \mathrm{~mL})$. The mixture was refluxed for $2 \mathrm{~h}$. The reaction was monitored by using TLC (hexane: ethyl acetate 2:8). After completion of the reaction, the mixture was cooled and the impurities were extracted with toluene. The aqueous phase was acidified with hydrochloric acid and the product was extracted with ethyl acetate $(20 \mathrm{~mL})$. The organic phase was washed with water, dried and evaporated to get compound 10. (0.42 g, 90\% yield). Off-white powder, M.p.: $110-111^{\circ} \mathrm{C}$. IR (KBr) $\mathrm{cm}^{-1}: 2919,2856,2537$, 1672, 1544, 1444, 1247, 1074, 830, 757. ${ }^{1} \mathrm{H}$ NMR $(400 \mathrm{MHz}$, DMSO- $\left._{6}\right) \delta 7.71(\mathrm{~s}, 1 \mathrm{H}), 7.22-7.17(\mathrm{~m}, 4 \mathrm{H}), 6.95-6.89(\mathrm{~m}$, $4 \mathrm{H}), 6.05-6.01(\mathrm{~m}, 2 \mathrm{H}), 5.42-5.38(\mathrm{~m}, 2 \mathrm{H}), 5.28-5.25(\mathrm{~m}$, 
2H), 4.56 (s, 4H). ${ }^{13} \mathrm{C}$ NMR (100 MHz, DMSO-d 6 ) $\delta 162.8$, $158.4,157.5,143.6,137.6,135.5,133.7,133.5,131.8,130.2$, $129.9,127.5,125.4,117.8,117.6,115.1,114.8,68.3,68.2$, 59.8, 20.8, 14.1. ESI-MS $m / z$ Calculated 392.1. Found: 391.4 $[\mathrm{M}-\mathrm{H}]^{-} .393 .2[\mathrm{M}+\mathrm{H}]^{+}$, Anal. Calculated for $\mathrm{C}_{23} \mathrm{H}_{20} \mathrm{O}_{4} \mathrm{~S}$ : C, 70.39; H, 5.14\%. Found: C, 70.42; H, 5.16\%.

2.2j Methyl 4,5-bis(4-(3-hydroxypropoxy)phenyl)thio phene-2-carboxylate (11): Methyl 4,5-bis(4-(allyloxy) phenyl)thiophene-2-carboxylate $(2 \mathrm{~g}, 0.005 \mathrm{~mol})$ dissolved in tetrahydrofuran $(50 \mathrm{~mL})$ and cooled to $0^{\circ} \mathrm{C}$. Borane $1 \mathrm{M}$ solution in THF $(11.8 \mathrm{~mL})$ was added and stirred at $0^{\circ} \mathrm{C}$ for $1 \mathrm{~h}$. The reaction mixture was slowly warmed to room temperature and maintained for $4 \mathrm{~h}$. The reaction mixture was quenched with water $(1 \mathrm{~mL})$ followed by addition of $2 \mathrm{~N}$ sodium hydroxide solution $(2 \mathrm{~mL})$, hydrogen peroxide $(40 \% \mathrm{w} / \mathrm{w}, 2 \mathrm{~mL})$ solution and stirred for $1 \mathrm{~h}$ at room temperature. The product was extracted with ethyl acetate $(100 \mathrm{~mL})$. The organic phase was separated and washed with $20 \%$ w/w sodium chloride solution followed by water, dried and evaporated under vacuum. The reaction produced a mixture of compounds, the major (80\%) component of the mixture being methyl 4,5-bis(4-(3-hydroxypropoxy)phenyl) thiophene-2-carboxylate $(\mathbf{1 1})\left(\mathrm{R}_{\mathrm{f}}=0.25, \mathrm{SiO}_{2}, 30 \%\right.$ ethyl acetate in hexanes), and the minor (20\%) methyl 5(4)(4-(2-hydroxypropoxy)phenyl)-4(5)-(4-(3-hydroxypropoxy) phenyl) thiophene-2-carboxylate $(\mathbf{1 2})\left(\mathrm{R}_{\mathrm{f}}=0.4, \mathrm{SiO}_{2}, 30 \%\right.$ ethyl acetate in hexanes). The product was isolated by column chromatography by eluting with $6: 4 \mathrm{v} / \mathrm{v}$ hexane-ethyl acetate to get compound $\mathbf{1 1}(1.2 \mathrm{~g}, 55 \%$ yield $)$ and $\mathbf{1 2}$ (0.3 g, 14\% yield). Yellow liquid, IR $(\mathrm{KBr}) \mathrm{cm}^{-1}: 3423$, 2928, 2877, 1712, 1547, 1446, 1247, 1063, 832, 755. ${ }^{1} \mathrm{H}$ NMR $\left(400 \mathrm{MHz}, \mathrm{CDCl}_{3}\right) \delta 7.77(\mathrm{~s}, 1 \mathrm{H}), 7.23(\mathrm{~d}, 2 \mathrm{H}, J=$ $8.76 \mathrm{~Hz}), 7.17(\mathrm{~d}, 2 \mathrm{H}, J=8.72 \mathrm{~Hz}), 6.83-6.80(\mathrm{~m}, 4 \mathrm{H}), 4.12$ $(\mathrm{t}, 4 \mathrm{H}, J=5.88 \mathrm{~Hz}), 3.89(\mathrm{~s}, 3 \mathrm{H}), 3.87-3.84(\mathrm{~m}, 4 \mathrm{H}), 2.06-$ $* 1.97(\mathrm{~m}, 4 \mathrm{H}) .{ }^{13} \mathrm{C}$ NMR $\left(100 \mathrm{MHz}, \mathrm{CDCl}_{3}\right) \delta 163.0,159.1$, 158.2, 145.2, 138.0, 136.5, 130.6, 130.3, 130.2, 128.4, 126.2, $114.7,114.6,65.7,65.6,60.5,60.3,52.3,32.1,29.8$. ESI-MS $\mathrm{m} / \mathrm{z}$ Calculated 442.2. Found: $487.2\left[\mathrm{M}+\mathrm{HCOO}^{-}\right.$. Anal. Calculated for $\mathrm{C}_{24} \mathrm{H}_{26} \mathrm{O}_{6} \mathrm{~S}: \mathrm{C}, 65.14 ; \mathrm{H}, 5.92 \%$. Found: $\mathrm{C}$, 65.16; H, 5.96\%.

2.2k Methyl5(4)-(4-(2-hydroxypropoxy)phenyl)-4(5)(4-(3-hydroxypropoxy)phenyl)thiophene-2-carboxylate (12): Yellow liquid, IR (KBr) $\mathrm{cm}^{-1}: 3400,2925,2872$, 1710, 1546, 1446, 1246, 1076, 830, 754. ${ }^{1} \mathrm{H}$ NMR $(400 \mathrm{MHz}$, $\left.\mathrm{CDCl}_{3}\right) \delta 7.78(\mathrm{~s}, 1 \mathrm{H}), 7.25-7.22(\mathrm{~m}, 2 \mathrm{H}), 7.19-7.16(\mathrm{~m}, 2 \mathrm{H})$, 6.84-6.82 (m, 4H), 4.20 (brs, $1 \mathrm{H}), 4.14(\mathrm{t}, 2 \mathrm{H}, J=8.76 \mathrm{~Hz})$, 3.96-3.94 (m, 1H), 3.90 (s, 3H), 3.89-3.87 (m, 2 H), 3.82$3.79(\mathrm{t}, 1 \mathrm{H}, J=8.32 \mathrm{~Hz}), 2.28-2.07(\mathrm{~m}, 1 \mathrm{H}), 2.06-2.04(\mathrm{~m}$, $2 \mathrm{H}), 1.70-1.65(\mathrm{~m}, 1 \mathrm{H}), 1.30(\mathrm{~d}, 3 \mathrm{H}) J=6.36 \mathrm{~Hz}) .{ }^{13} \mathrm{C}$ NMR $\left(100 \mathrm{MHz}, \mathrm{CDCl}_{3}\right) \delta 163.0,158.8,158.2,145.2,138.1$, 136.6, 130.7, 130.5, 130.4, 130.3, 128.4, 126.2, 114.8, 114.7, 73.4, 66.4, 65.8, 60.6, 52.4, 32.1, 18.92. ESI-MS m/z Calculated 442.2. Found: $487.3\left[\mathrm{M}+\mathrm{HCOO}^{-}\right.$. Anal. Calculated for $\mathrm{C}_{24} \mathrm{H}_{26} \mathrm{O}_{6} \mathrm{~S}: \mathrm{C}, 65.14 ; \mathrm{H}, 5.92 \%$. Found: $\mathrm{C}, 65.18 ; \mathrm{H}$, $5.94 \%$.
2.21 Ethyl 4,5-bis(4-(3-hydroxypropoxy)phenyl)thio phene-2-carboxylate (13): Ethyl 4,5-bis(4-(allyloxy) phenyl)thiophene-2-carboxylate $(2.1 \mathrm{~g}, 0.005 \mathrm{~mol})$ was dissolved in tetrahydrofuran $(50 \mathrm{~mL})$ and cooled to $0^{\circ} \mathrm{C}$. Borane $1 \mathrm{M}$ solution in THF $(11.8 \mathrm{~mL})$ was added and stirred at $0^{\circ} \mathrm{C}$ for $1 \mathrm{~h}$. The reaction mixture was slowly warmed to room temperature and maintained for $4 \mathrm{~h}$. The reaction mixture was quenched with water $(1 \mathrm{~mL})$ followed by addition of $2 \mathrm{~N}$ sodium hydroxide solution $(2 \mathrm{~mL})$, hydrogen peroxide $(40 \% \mathrm{w} / \mathrm{w}, 2 \mathrm{~mL})$ solution and stirred for $1 \mathrm{~h}$ at room temperature. The product was extracted with ethyl acetate $(100 \mathrm{~mL})$ and the organic phase was separated, washed with $20 \% \mathrm{w} / \mathrm{w}$ sodium chloride solution followed by water, dried and evaporated under vacuum. The reaction produced a mixture of compounds, the major (80\%) component of the mixture being ethyl 4,5-bis(4-(3-hydroxypropoxy)phenyl)thiophene2-carboxylate $(\mathbf{1 3})\left(\mathrm{R}_{f}=0.25, \mathrm{SiO}_{2}, 30 \%\right.$ ethyl acetate in hexanes), and the minor (20\%) ethyl 5(4)(4-(2-hydroxypropoxy)phenyl)-4(5)-(4-(3-hydroxypropoxy) phenyl)thiophene-2-carboxylate (14) $\left(\mathrm{R}_{\mathrm{f}}=0.4, \mathrm{SiO}_{2}, 30 \%\right.$ ethyl acetate in hexanes). The product was isolated by column chromatography by eluting with $6: 4 \mathrm{v} / \mathrm{v}$ hexane-ethyl acetate to get compound $\mathbf{1 3}$ (1.3 g, 57\% yield) and $\mathbf{1 4}$ (0.4 g, 17\% yield). Yellow liquid, IR $(\mathrm{KBr}) \mathrm{cm}^{-1}: 3413$, 2926, 2874, 1704, 1547, 1443, 1246, 1061, 830, 754. ${ }^{1} \mathrm{H}$ NMR $\left(400 \mathrm{MHz}, \mathrm{CDCl}_{3}\right) \delta 7.78(\mathrm{~s}, 1 \mathrm{H}), 7.25(\mathrm{~d}, 2 \mathrm{H}, J=$ $8.8 \mathrm{~Hz}), 7.18(\mathrm{~d}, 2 \mathrm{H}, J=8.72 \mathrm{~Hz}), 6.85-6.82(\mathrm{~m}, 4 \mathrm{H}), 4.40$ (q, $2 \mathrm{H}, J=7.12 \mathrm{~Hz}), 3.89$ (m, 4H), 2.85 (s, 2H), 2.08-1.98 (m, 4H), 1.44-1.38(m, 3H). $\left.{ }^{13} \mathrm{C} \mathrm{NMR} \mathrm{(100} \mathrm{MHz,} \mathrm{CDCl}_{3}\right) \delta$ 162.6, 159.1, 158.2, 144.9, 137.9, 136.3, 130.9, 130.6, 130.3, $128.5,126.2,114.7,114.6,65.8,65.7,61.3,60.4,60.3,32.2$, 32.1, 14.5. ESI-MS $\mathrm{m} / z 501.3\left[\mathrm{M}+\mathrm{HCOO}^{-}\right.$. ESI-MS $\mathrm{m} / z$ Calculated 456.2. Found: $501.3\left[\mathrm{M}+\mathrm{HCOO}^{-}\right.$. Anal. Calculated for $\mathrm{C}_{25} \mathrm{H}_{28} \mathrm{O}_{6} \mathrm{~S}$ : C, 65.77; $\mathrm{H}, 6.18 \%$. Found: $\mathrm{C}, 65.74$; $\mathrm{H}, 6.16 \%$.

2.2m Ethyl 5(4)-(4-(2-hydroxypropoxy)phenyl)-4(5)(4-(3-hydroxypropoxy)phenyl)thiophene-2-carboxylate (14): Yellow liquid, IR (KBr) $\mathrm{cm}^{-1}: 3421,2926,2873$, $1705,1547,1443,1247,1072,830,754 .{ }^{1} \mathrm{H}$ NMR $(400 \mathrm{MHz}$, $\left.\mathrm{CDCl}_{3}\right) \delta 7.70(\mathrm{~s}, 1 \mathrm{H}), 7.17-7.14(\mathrm{~m}, 2 \mathrm{H}), 7.10-7.08(\mathrm{~m}$, $2 \mathrm{H})$, 6.77-6.73 (m, 4H), 4.31-4.27 (m, 2H), 4.14-4.10 $(\mathrm{m}, 1 \mathrm{H}), 4.06(\mathrm{t}, 2 \mathrm{H}, J=5.28 \mathrm{~Hz}), 3.88-3.84(\mathrm{~m}, 1 \mathrm{H})$, $3.81-3.77(\mathrm{~m}, 2 \mathrm{H}), 3.74(\mathrm{t}, 1 \mathrm{H}, J=8.28 \mathrm{~Hz}), 1.99-$ $1.96(\mathrm{~m}, 2 \mathrm{H}), 1.35-1.33(\mathrm{~m}, 3 \mathrm{H}), 1.22-1.20(\mathrm{~m}, 3 \mathrm{H}) .{ }^{13} \mathrm{C}$ NMR $\left(100 \mathrm{MHz}, \mathrm{CDCl}_{3}\right) \delta 161.4,157.9,157.0,143.9,136.9$, 135.0, 129.8, 129.5, 129.2, 127.6, 125.4, 113.7, 113.5, 72.2, 65.2, 64.6, 60.2, 59.4, 30.9, 17.6, 13.4. ESI-MS m/z Calculated 456.2. Found: $501.2\left[\mathrm{M}+\mathrm{HCOO}^{-}\right.$. Anal. Calculated for $C_{25} \mathrm{H}_{28} \mathrm{O}_{6} \mathrm{~S}: \mathrm{C}, 65.77 ; \mathrm{H}, 6.18 \%$. Found: $\mathrm{C}, 65.72 ; \mathrm{H}$, $6.14 \%$.

\section{2n 4,5-Bis(4-(3-hydroxypropoxy)phenyl)thiophene- 2-carboxylic acid (15): Methyl 4,5-bis(4- (3-hydroxypropoxy)phenyl)thiophene-2-carboxylate ( $0.5 \mathrm{~g}$, $0.001 \mathrm{~mol}$ ) was added at room temperature to a solution of sodium hydroxide $(0.5 \mathrm{~g}, 0.0125 \mathrm{~mol})$ in ethanol:water}


$(8 \mathrm{~mL}: 2 \mathrm{~mL})$. The mixture was refluxed for $2 \mathrm{~h}$. The reaction was monitored by TLC using hexane:ethyl acetate $(2 \mathrm{~mL}: 8 \mathrm{~mL})$ as a solvent system. After the reaction was completed, the reaction mixture was cooled and the impurities were extracted with toluene. The aqueous phase was acidified with hydrochloric acid and the product was extracted with ethyl acetate $(20 \mathrm{~mL})$. The organic phase was washed with water, dried and evaporated to get compound 15. (0.44 g, $91 \%$ yield). Pale brown powder, M.p.: $136-138^{\circ} \mathrm{C}$. IR $(\mathrm{KBr})$ $\mathrm{cm}^{-1}: 3385,2925,2854,2602,1686,1547,1442,1248,1086$, 832, 757. ${ }^{1} \mathrm{H}$ NMR $\left(400 \mathrm{MHz}, \mathrm{DMSO}-\mathrm{d}_{6}\right) \delta 7.71(\mathrm{~s}, 1 \mathrm{H})$, $7.23(\mathrm{~d}, 2 \mathrm{H}, J=8.64 \mathrm{~Hz}), 7.17(\mathrm{~d}, 2 \mathrm{H}, J=8.6 \mathrm{~Hz}), 6.93$ $(\mathrm{d}, 2 \mathrm{H}, J=8.76 \mathrm{~Hz}), 6.90(\mathrm{~d}, 2 \mathrm{H}, J=8.72 \mathrm{~Hz}), 4.05$ (quartet, $4 \mathrm{H}, J=6.0 \mathrm{~Hz}), 3.58(\mathrm{t}, 4 \mathrm{H}, J=6.08 \mathrm{~Hz}), 1.90$ (quintet, $4 \mathrm{H}, J=6.12 \mathrm{~Hz}) .{ }^{13} \mathrm{C}$ NMR $\left(100 \mathrm{MHz}, \mathrm{DMSO}-\mathrm{d}_{6}\right) \delta 162.8$, 158.9, 158.0, 143.6, 137.6, 131.7, 130.2, 129.9, 127.3, 125.1, 114.8, 114.5, 114.3, 64.6, 64.5, 57.2, 32.1, 32.0. ESI-MS m/z Calculated 428.1. Found: 427.2[M-H] ${ }^{-}$. Anal. Calculated for $\mathrm{C}_{23} \mathrm{H}_{24} \mathrm{O}_{6} \mathrm{~S}: \mathrm{C}, 64.47 ; \mathrm{H}, 5.65 \%$. Found: C, 64.49; H, 5.62\%.

2.2o 5(4)-(4-(2-Hydroxypropoxy)phenyl)-4(5)-(4-(3hydroxypropoxy)phenyl)thiophene-2-carboxylic acid (16): Methyl 5(4)-(4-(2-hydroxypropoxy)phenyl)-4(5)-(4(3-hydroxypropoxy)phenyl)thiophene-2-carboxylate $(0.5 \mathrm{~g}$, $0.001 \mathrm{~mol}$ ) was added at room temperature to a solution of sodium hydroxide $(0.5 \mathrm{~g}, 0.0125 \mathrm{~mol})$ in ethanol:water $(8 \mathrm{~mL}: 2 \mathrm{~mL})$. The mixture was refluxed for $2 \mathrm{~h}$. The reaction was monitored by using TLC (hexane:ethyl acetate 2:8). After the reaction was completed, the reaction mixture was cooled and the impurities were extracted with toluene. The aqueous phase was acidified with hydrochloric acid and the product was extracted with ethyl acetate $(20 \mathrm{~mL})$. The organic phase was washed with water, dried and evaporated to get compound 16. $\left(0.42 \mathrm{~g}, 87 \%\right.$ yield). Yellow powder, M.p.: $150-152^{\circ} \mathrm{C}$. IR (KBr) $\mathrm{cm}^{-1}: 3394,2923,2853,1682,1545,1445,1246$, $1060,830,754 .{ }^{1} \mathrm{H}$ NMR $(400 \mathrm{MHz}$, DMSO-d6) $\delta 12.9$ (brs, $1 \mathrm{H}), 7.71(\mathrm{~s}, 1 \mathrm{H}), 7.22-7.17(\mathrm{~m}, 4 \mathrm{H}), 6.93-6.87(\mathrm{~m}, 4 \mathrm{H}), 4.90$ $(\mathrm{s}, 1 \mathrm{H}), 4.58(2,1 \mathrm{H}), 4.05-4.01(\mathrm{~m}, 2 \mathrm{H}), 3.97-3.93(\mathrm{~m}, 1 \mathrm{H})$, $3.85-3.76(\mathrm{~m}, 2 \mathrm{H}), 3.57-3.55(\mathrm{~m}, 2 \mathrm{H}), 1.89-1.83(\mathrm{~m}, 2 \mathrm{H})$, $1.16(\mathrm{t}, 3 \mathrm{H}, J=6.24 \mathrm{~Hz}) .{ }^{13} \mathrm{C} \mathrm{NMR}\left(100 \mathrm{MHz}, \mathrm{DMSO}-\mathrm{d}_{6}\right) \delta$ 162.8, 158.9, 158.0, 143.6, 137.6, 131.8, 130.2, 129.9, 127.3, $127.2,125.2,125.1,115.1,114.9,114.8,114.5,114.2,64.8$, 64.5, 57.2, 32.1, 20.1. ESI-MS $\mathrm{m} / \mathrm{z}$ Calculated 428.1. Found: $427.2[\mathrm{M}-\mathrm{H}]^{-}$. Anal. Calculated for $\mathrm{C}_{23} \mathrm{H}_{24} \mathrm{O}_{6} \mathrm{~S}: \mathrm{C}, 64.47$; H, 5.65\%. Found: C, 64.45; H, 5.68\%.

\subsection{In vitro anti-inflammatory activity (anti-denaturation assay)}

The in vitro anti-inflammatory activity of synthesized compounds were studied using bovine serum albumin denaturation method. ${ }^{17,18}$ In brief, increasing concentrations of the test or reference compound were incubated with $0.5 \%$ w/v of bovine serum albumin at $37^{\circ} \mathrm{C}$ for $20 \mathrm{~min}$ and the temperature was increased to keep the samples at $57^{\circ} \mathrm{C}$ for $30 \mathrm{~min}$. After cooling to room temperature, the turbidity was measured using UV-Visible spectrophotometer at $660 \mathrm{~nm}$ following addition of phosphate buffered saline. The control represents $100 \%$ protein denaturation. The results were compared with reference drug diclofenac sodium. The percentage inhibition of protein denaturation was calculated by using the following formula:

$$
\begin{aligned}
& \text { Percentage Inhibition }=100 \\
& -[\text { (optical density of test solution } \\
& \quad-\text { optical density of product control }) \\
& \quad \div \text { (optical density of test control })] \times 100 .
\end{aligned}
$$

\subsection{In vitro anti-oxidant activity}

The antioxidant activity of the test drug was determined using the 1,1-diphenyl-2 picrylhydrazyl (DPPH) free radical scavenging assay. ${ }^{19}$ The test drug was mixed with $95 \%$ methanol to prepare the stock solution in required concentration $(100 \mu \mathrm{g} / \mathrm{mL})$. From the stock solution, $10 \mu \mathrm{g} / \mathrm{mL}, 20 \mu \mathrm{g} / \mathrm{mL}$, $40 \mu \mathrm{g} / \mathrm{mL}, 60 \mu \mathrm{g} / \mathrm{mL}$ and $100 \mu \mathrm{g} / \mathrm{mL}$ concentration of test drug was prepared. Ascorbic acid was used as standard and was prepared in same concentration as that of the test drug by using methanol as solvent. Final reaction mixture containing $1 \mathrm{~mL}$ of $0.3 \mathrm{mmol} \mathrm{DPPH}$ methanol solution was added to $2.5 \mathrm{~mL}$ of sample solution of different concentrations and allowed to react at room temperature after $15 \mathrm{~min}$ incubation period at $37^{\circ} \mathrm{C}$. Absorbance was read out at $517 \mathrm{~nm}$. Control reading was observed without adding test drug. \% scavenging $=[$ (Absorbance of control - Absorbance of test sample $) \div$ (Absorbance of control) $] \times 100$.

\subsection{Molecular docking studies}

Molecular docking was performed for the synthesised compounds in order to investigate their possible binding mode in the cyclooxygenase-2 (COX-2) receptor. Docking was performed using the Auto Dock Tools (ADT) version 1.5.6 and Auto Dock version 4.2.5.1 docking program. ${ }^{20,21}$ The structure of COX-2 was downloaded from the Protein Data Bank (PDB ID: 1PXX). ${ }^{22}$ The co-crystallized ligand and water molecules present in the 1PXX structure were removed. Then, polar hydrogen atoms were added, and lower occupancy residue structures were deleted. ADT was used to replace any incomplete side chains. Gasteiger charges were added to each atom and merged the non-polar hydrogen atoms to the protein structure. The hydrogen bond distance between donor and acceptor atoms were defined as $1.9 \AA$ with a tolerance of $0.5 \AA$ and the threshold for acceptor-hydrogen-donor angle was set to not less than $120^{\circ}$. For further studies in ADT, the structures were saved in PDBQT file format. A grid box centred on $27.131,24.348$ and 14.747 with the dimension of $60 \times 60 \times 60 \AA^{3}$ and $0.375 \AA$ spacing was created around the binding site of co-crystallised ligand using ADT. Grid energy calculations were carried out with the centre of the box was set at co-crystallised ligand centre. Default docking parameters were used and twenty docked conformations for each compound were generated. Genetic algorithm was used to estimate the energy of the binding interactions. PyMOL 
was used to visualise the binding modes and interactions of the docked compounds with amino acid residues in the active site of COX-2 receptor.

\section{Results and Discussion}

\subsection{Chemistry}

In the present work, we synthesized a new series of allyloxy and hydroxypropoxy derivatives of 4,5-diaryl thiophene-2-carboxylic acid, as shown in Scheme 1. The first step in the Scheme 1 is the Vilsmeier reaction $^{23,24}$ of desoxyanisoin $\mathbf{1}$ with dimethylformamide (DMF) and phosphorous oxychloride $\left(\mathrm{POCl}_{3}\right)$ to give compound $\mathbf{2}$ in $78 \%$ yield after recrystallization from ethanol. ${ }^{12}$ Compound $\mathbf{2}$ was condensed and cyclized with 2-mercaptoacetic acid (thioglycolic acid) in the presence of potassium hydroxide to afford compound $\mathbf{3}^{25}$ in $71 \%$ yield. Treatment of compound 3 with aluminium chloride in chlorobenzene ${ }^{16,26}$ at $95-105^{\circ} \mathrm{C}$ over $3 \mathrm{~h}$ under stirring is vital for the demethylation of compound $\mathbf{3}$ to get compound $\mathbf{4}$ in good yield (88\%). The obtained compound $\mathbf{4}$ did not require any further purification.

The compound 4 obtained was converted to the corresponding ester $\mathbf{5}$ and $\mathbf{6}$ by using alcohol in presence of sulphuric acid. Esterification of compound 4 with methanol or ethanol and sulphuric acid under reflux condition over $8 \mathrm{~h}$ gave $86 \%$ and $88 \%$ yield of compound 5 and6, respectively. The allyation of compound 4,5 and 6 with ally bromide and potassium carbonate ${ }^{27}$ in acetonitrile under reflux condition gave $87 \%, 88 \%$ and $82 \%$, yield of compound 7,8 and 9 , respectively.

The allyl carboxylic acid ester $\mathbf{7}$ or $\mathbf{8}$ or $\mathbf{9}$ was hydrolysed with sodium hydroxide to get compound $\mathbf{1 0}$ in $90 \%$ yield. Similarly compound 14 was hydrolysed with sodium hydroxide to get carboxylic acid compound $\mathbf{1 6}$ in $88 \%$ yield.

The allyl carboxylic acid ester 7 was converted to alcohol by hydroboration followed by oxidation with hydrogen peroxide and sodium hydroxide solution. ${ }^{27}$ The reaction produced a mixture of compounds, the major $(80 \%)$ component of the mixture being methyl 4,5-bis(4-(3-hydroxypropoxy)phenyl)thiophene2-carboxylate compound 11 and the minor (20\%) methyl 5(4)-(4-(2-hydroxypropoxy)phenyl)-4(5)-(4-(3hydroxypropoxy)phenyl)thiophene-2-carboxylate compound 12. The product was isolated by column chromatography eluting with $6: 4 \mathrm{v} / \mathrm{v}$ hexane-ethyl acetate to afford the product 11 (55\% yield) and 12 (14\% yield).

Similarly compound $\mathbf{8}$ was converted to alcohol compound $\mathbf{1 3}$ (57\% yield) and $\mathbf{1 4}$ (17\% yield). The carboxylic acid ester $\mathbf{1 1}$ or $\mathbf{1 3}$ was hydrolysed with sodium hydroxide in aqueous ethanol under reflux condition to get compound $\mathbf{1 5}$ in $88 \%$ yields. Similarly compound 12 and 14 was hydrolysed with sodium hydroxide in aqueous ethanol under reflux condition to get compound 16 in $88 \%$ yield.

\subsection{Characterization}

The structure of the synthesized intermediate compound 7 was confirmed by various spectral techniques such as NMR, Mass and IR data. IR spectrum of the compound 7 revealed that the bands between $\sim 2950 \mathrm{~cm}^{-1}$ and $\sim 2850 \mathrm{~cm}^{-1}$ correspond to the asymmetric and symmetric stretching of methylene groups and the band at around $\sim 1704 \mathrm{~cm}^{-1}$ corresponds to the carbonyl group of the ester moiety. The ${ }^{1} \mathrm{H}$ NMR spectrum recorded in $\mathrm{CDCl}_{3}$ showed twenty-two protons. The singlet observed at $\delta 7.78 \mathrm{ppm}$ corresponds to the aryl proton of the thiophene moiety. The multiplet at around $\delta$ 4.54-4.52 ppm corresponds to the methylene protons attached to phenoxy ring system. The singlet observed at $\delta 3.89 \mathrm{ppm}$ corresponds to the methyl proton of the ester group. The ${ }^{13} \mathrm{C}$ NMR spectrum recorded in $\mathrm{CDCl}_{3}$ showed twenty-four signals, in which the signal observed at $\delta 162.9 \mathrm{ppm}$ correspond to carbonyl carbon of the ester moiety. The two signals at $\delta 114.9 \mathrm{ppm}$ and at $\delta 114.8 \mathrm{ppm}$ correspond to the four aryl carbons at the ortho position to hydroxyl derivative of the phenyl rings. The signals at $\delta 68.9$ and $\delta 68.8 \mathrm{ppm}$ confirmed the methylene carbons of allyloxy group. The signal at $\delta 52.3 \mathrm{ppm}$ confirmed the methyl carbon of ester group. Mass spectrum acquired in positive ionization ESI mode, showed a signal at 407.1 Da corresponding to $[\mathrm{M}+\mathrm{H}]^{+}$, which confirmed the molecular mass of the compound. In addition to the above spectral evidences discussed, the structure of synthesized compound 7 was further confirmed unequivocally with single-crystal Xray diffraction data. The crystal data was deposited at CCDC, and the CCDC No. is 1406560 . The ORTEP diagram of above crystal compound is shown in Figure 2.

The structure of the synthesized compound $\mathbf{1 5}$ was confirmed by various spectral techniques such as NMR, Mass and IR data. IR spectrum of the compound 15 revealed that the broad band observed at around $3360-3300 \mathrm{~cm}^{-1}$ corresponds to the carboxylic acid stretching coupled with the hydroxyl stretching of the propyl group. The bands observed at $\sim 2925 \mathrm{~cm}^{-1}$ and $\sim 2854 \mathrm{~cm}^{-1}$ correspond to the asymmetric and symmetric stretching of methylene groups. The band at around $\sim 1686 \mathrm{~cm}^{-1}$ corresponds to the carbonyl group of the acid moiety. The ${ }^{1} \mathrm{H}$ NMR spectrum recorded in DMSO- $\mathrm{d}_{6}$ showed twenty-four protons in which the protons of hydroxyl and carboxylic groups weren't 

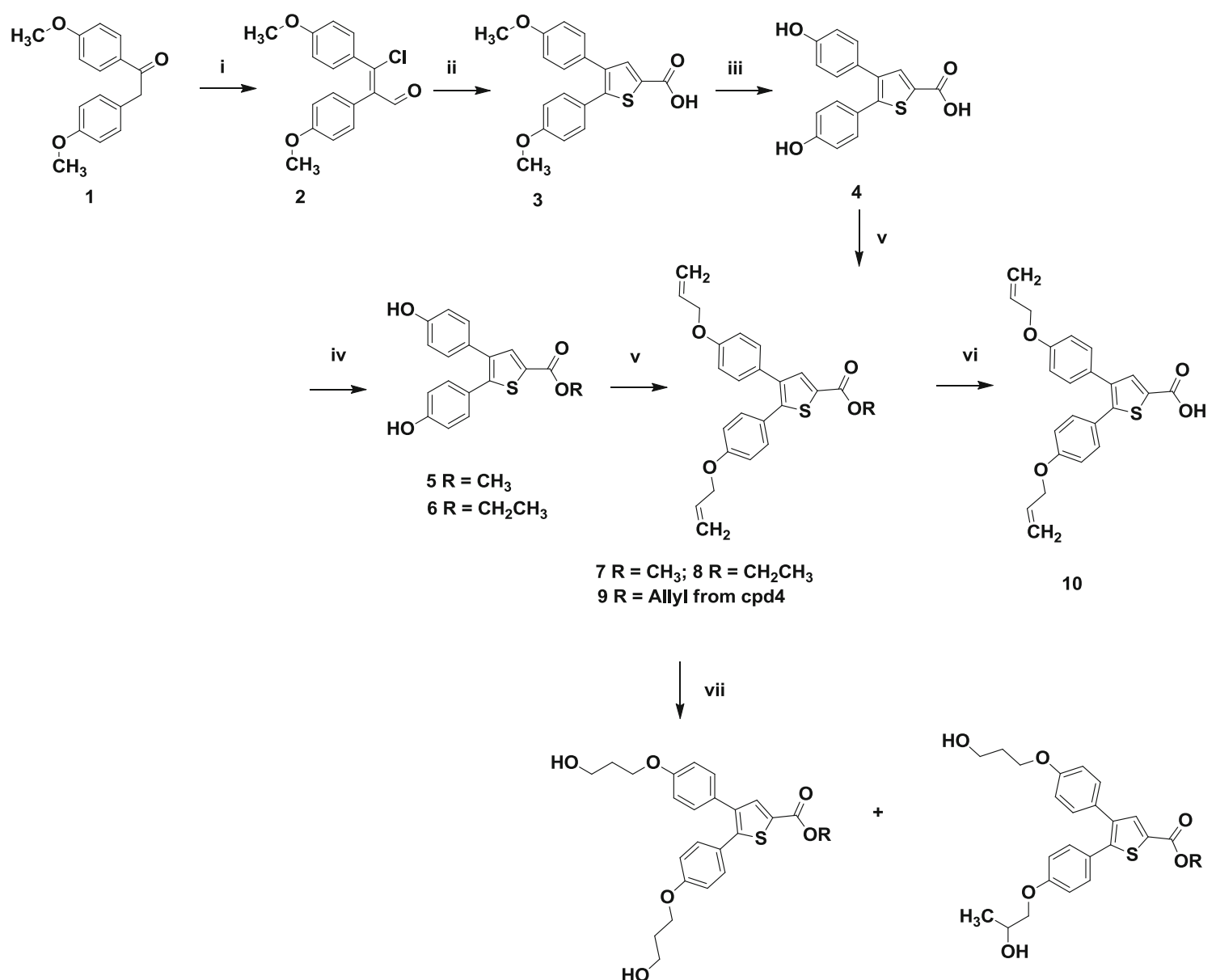

$11 \mathrm{R}=\mathrm{CH}_{3} ; 13 \mathrm{R}=\mathrm{CH}_{2} \mathrm{CH}_{3}$

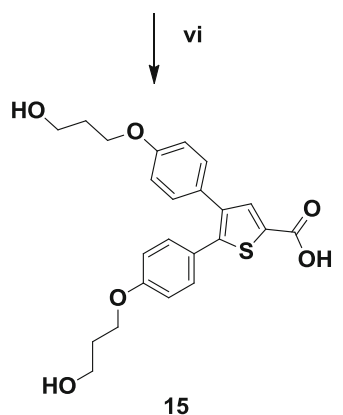

$12 \mathrm{R}=\mathrm{CH}_{3} ; 14 \mathrm{R}=\mathrm{CH}_{2} \mathrm{CH}_{3}$

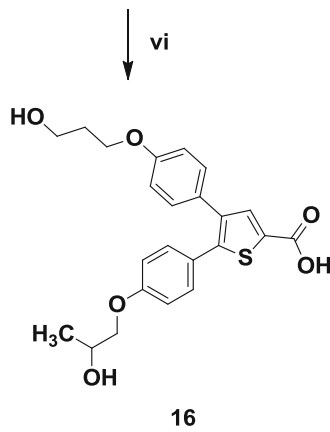

Scheme 1. Synthesis of 4,5-diarylthiophene-2-carboxylic acid derivatives. Reagents and conditions: (i) $\mathrm{DMF}$ and $\mathrm{POCl}_{3}$, at $70-75^{\circ} \mathrm{C}$ for $4 \mathrm{~h}$; (ii) 2-mercapto acetic acid, $\mathrm{KOH}$, methanol and water, at reflux for $4 \mathrm{~h}$; (iii) $\mathrm{AlCl}_{3}$ and chlorobenzene, at $95-105^{\circ} \mathrm{C}$ for $3 \mathrm{~h}$; (iv) methanol or ethanol, sulphuric acid, at reflux for $8 \mathrm{~h}$; (v) allyl bromide, $\mathrm{K}_{2} \mathrm{CO}_{3}$, acetonitrile reflux for $4 \mathrm{~h}$; (vi) $\mathrm{NaOH}$, ethanol and water, at reflux for $2 \mathrm{~h}$; (vii) Borane in $\mathrm{THF}$ at $0^{\circ} \mathrm{C} 1 \mathrm{~h}, \mathrm{H}_{2} \mathrm{O}_{2} / \mathrm{NaOH}$ at $\mathrm{RT} 1 \mathrm{~h}$.

observed. The singlet observed at $\delta 7.71 \mathrm{ppm}$ corresponds to the aryl proton of the thiophene ring system. The quartet at $\delta 4.05 \mathrm{ppm}$ corresponds to four methylene protons attached to two hydroxy groups, the triplet at $\delta 3.58 \mathrm{ppm}$ corresponds to four methylene protons attached to two phenoxy group. The ${ }^{13} \mathrm{C}$ NMR spectrum recorded in DMSO- $\mathrm{d}_{6}$ showed twenty-three signals, in which the signal observed at $\delta 162.9 \mathrm{ppm}$ correspond to carbonyl carbon of the acid group. The four signals at $\delta 114.9, \delta 114.8, \delta 114.7$ and $\delta 114.5 \mathrm{ppm}$ correspond to the four aryl carbons of the phenoxy ring. The signal at $\delta 57.5 \mathrm{ppm}$ corresponds to the methylene groups of the hydroxy propyl side chain. Mass spectrum acquired in negative ionization ESI mode showed the signal at 427.2 Da which corresponds to $[\mathrm{M}-\mathrm{H}]$ confirmed the molecular mass of the compound. 


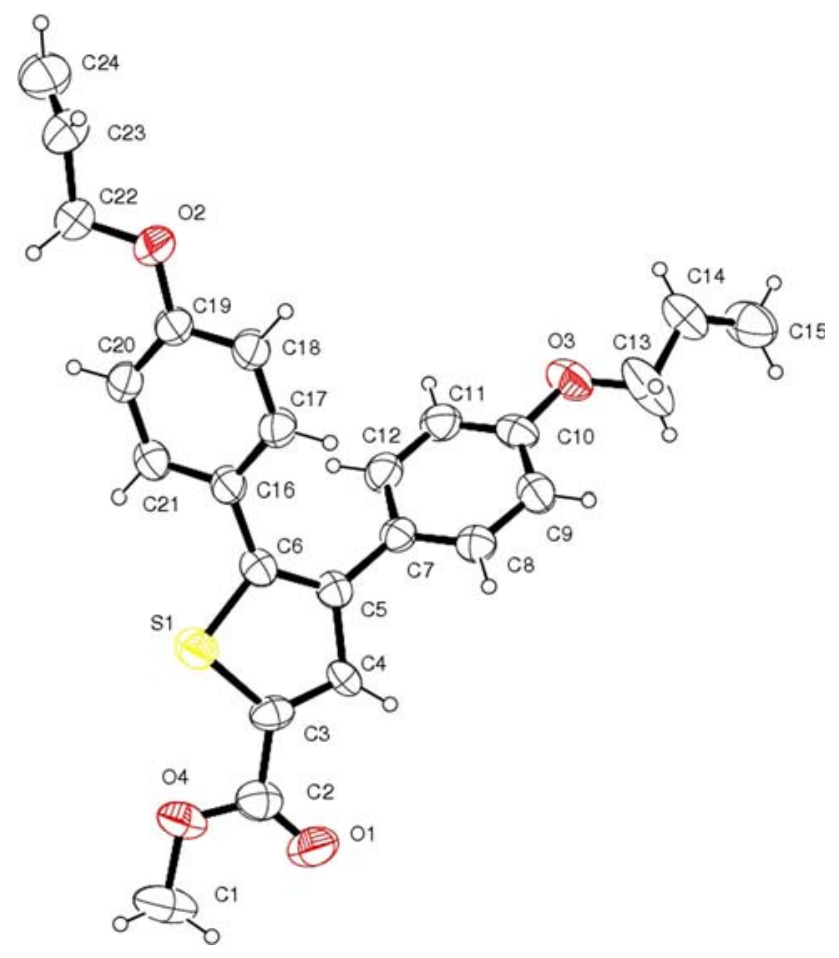

Figure 2. ORTEP diagram of compound 7 (methyl 4,5-bis(4-(allyloxy)phenyl)thiophene-2-carboxylate, CCDC 1406560).

\subsection{Biological evaluation: structure activity relationship (SAR)}

3.3a In vitro anti-inflammatory activity: In our earlier studies, we have identified that the introduction of amino moieties at carboxylic acid of thiophene ring ${ }^{16}$ significantly enhanced the anti-inflammatory activity spectrum of 4,5-diarylthiophene-2-carboxylic acid analogues. In the present study, we developed and synthesized some newer derivatives of compound 4 by introducing hydroxyl substituents to the phenyl ring and esterification of carboxylic acid of thiophene ring. The anti-inflammatory activity of novel substituted 4,5-diarylthiophene-2-carboxylic acid derivatives were evaluated using inhibition of bovine serum albumin denaturation method and compared with standard drug diclofenac sodium. The results are summarized in Table 1.

Most of the derivatives exhibited anti-inflammatory activity that is comparable to diclofenac sodium. The free acid $\mathbf{4}$ is less potent; however, derivatisation of the hydroxy group to allyl with bisallyloxy substituted phenyl moiety (10) resulted in analogues having antiinflammatory activity comparable to the standard drug. Although, bisallyloxy substituted phenyl moiety with methyl (7), ethyl (8) and allyl (9) esters of acid group resulted in less active analogues. In contrast, the compound 4,5-bis(4-(3-hydroxypropoxy)phenyl)thiophene2-carboxylic acid (15) and its methyl (11), or ethyl ester (13) exhibited better anti-inflammatory activity in comparison to the standard drug whereas their byproducts 5(4)-(4-(2-hydroxypropoxy)phenyl)-4(5)-(4-(3hydroxypropoxy)phenyl)thiophene-2-carboxylic acid (16) and its methyl (12) or ethyl ester (14) showed moderate activity.

Taken together, these results clearly suggest that allyloxy/hydroxypropoxy substitution on the phenyl group of 4,5-diarylthiophene-2-carboxylic acid derivatives is advantageous in improving or retaining the anti-inflammatory spectrum. Further, an important SAR observation relevant to the nature of substituents to the diaryl ring indicates that symmetricity of substituents is

Table 1. In vitro anti-inflammatory activity of compounds 4, 7-16 by inhibition of protein denaturation method (Bovine serum albumin).

\begin{tabular}{lllllll}
\hline \multirow{2}{*}{ Entry } & Compounds & \multicolumn{5}{c}{ Activity (\% inhibition of protein denaturation) } \\
\cline { 3 - 7 } & & $25 \mu \mathrm{g} / \mathrm{mL}$ & $50 \mu \mathrm{g} / \mathrm{mL}$ & $100 \mu \mathrm{g} / \mathrm{mL}$ & $200 \mu \mathrm{g} / \mathrm{mL}$ & $400 \mu \mathrm{g} / \mathrm{mL}$ \\
\hline 1 & $\mathbf{4}$ & 4.86 & 9.84 & 16.47 & 28.13 & 30.33 \\
2 & $\mathbf{7}$ & 10.42 & 18.85 & 26.04 & 35.41 & 43.75 \\
3 & $\mathbf{8}$ & 5.21 & 12.5 & 17.71 & 21.88 & 29.17 \\
4 & $\mathbf{9}$ & 14.58 & 20.83 & 29.17 & 35.42 & 41.67 \\
5 & $\mathbf{1 0}$ & 34.38 & 42.71 & 51.05 & 62.5 & 70.83 \\
6 & $\mathbf{1 1}$ & 22.92 & 31.25 & 45.83 & 55.21 & 60.42 \\
7 & $\mathbf{1 2}$ & 19.79 & 29.17 & 38.54 & 46.88 & 52.08 \\
8 & $\mathbf{1 3}$ & 35.42 & 40.63 & 50.00 & 62.50 & 75.00 \\
9 & $\mathbf{1 4}$ & 14.58 & 23.96 & 31.25 & 46.88 & 54.17 \\
10 & $\mathbf{1 5}$ & 21.88 & 34.38 & 43.75 & 57.3 & 70.63 \\
11 & $\mathbf{1 6}$ & 8.33 & 15.63 & 22.92 & 31.29 & 45.83 \\
12 & Std & 32.29 & 40.65 & 51.04 & 60.41 & 66.66 \\
\hline
\end{tabular}

Std: Diclofenac sodium 
Table 2. Antioxidant activity of compounds 4, 7-16 by using 1,1-diphenyl-2-picrylhydrazyl (DPPH) free radical scavenging assay.

\begin{tabular}{lcclllll}
\hline \multirow{2}{*}{ Entry } & \multirow{2}{*}{ Compounds } & \multicolumn{5}{c}{$\%$ Inhibition } & $\begin{array}{l}\text { Antioxidant } \\
\text { IC50 value }\end{array}$ \\
\cline { 3 - 6 } & & $10 \mu \mathrm{g} / \mathrm{mL}$ & $20 \mu \mathrm{g} / \mathrm{mL}$ & $40 \mu \mathrm{g} / \mathrm{mL}$ & $60 \mu \mathrm{g} / \mathrm{mL}$ & $100 \mu \mathrm{g} / \mathrm{mL}$ & \\
\hline 1 & $\mathbf{4}$ & 3.59 & 7.61 & 14.16 & 19.34 & 23.78 & 208.64 \\
2 & $\mathbf{7}$ & 2.78 & 3.17 & 9.83 & 12.68 & 19.45 & 255.54 \\
3 & $\mathbf{8}$ & 6.76 & 15.01 & 17.34 & 27.17 & 30.97 & 164.96 \\
4 & $\mathbf{9}$ & 1.27 & 8.77 & 13.53 & 18.39 & 23.78 & 204.97 \\
5 & $\mathbf{1 0}$ & 24.1 & 30.87 & 39.22 & 46.93 & 53.28 & 80.95 \\
6 & $\mathbf{1 1}$ & 21.25 & 28.86 & 37.42 & 43.66 & 50.63 & 89.48 \\
7 & $\mathbf{1 2}$ & 9.62 & 15.12 & 20.82 & 27.91 & 34.88 & 148.87 \\
8 & $\mathbf{1 3}$ & 27.27 & 39.32 & 45.77 & 52.75 & 65.22 & 56.16 \\
9 & $\mathbf{1 4}$ & 13.95 & 19.45 & 24.84 & 28.86 & 37.53 & 146.81 \\
10 & $\mathbf{1 5}$ & 9.73 & 13.11 & 23.57 & 32.03 & 37.42 & 129.19 \\
11 & $\mathbf{1 6}$ & 12.05 & 24.63 & 29.07 & 35.41 & 41.23 & 120.77 \\
12 & Std & 41.01 & 61.84 & 73.25 & 86.89 & 92.39 & 5.754 \\
\hline
\end{tabular}

Std: Ascorbic acid

required for optimal anti-inflammatory as in case of $\mathbf{1 1}$ and $\mathbf{1 5}$ whereas when the symmetricity is lost $(\mathbf{1 2}, \mathbf{1 4}$ and 16) a reduction in activity was observed. This indicates that lengthier substituents to the diaryl ring are well tolerated resulting in substantial increase of antiinflammatory potency.

The 4,5-diarylthiophene-2-carboxylic acid 4 or its carboxyamide analogue reported in our earlier work ${ }^{16}$ possess very little anti-inflammatory property in comparison to diclofenac. On the other hand, derivatization of the acid to ester or the amide with $p$-halo substituted phenyl moiety resulted in analogues possessing activity comparable to diclofenac. The lipophilic substituent, namely the hydroxypropyl moiety to the diaryl ring as in $\mathbf{1 3}$ and $\mathbf{1 5}$ appears to be the most important substituent with regard to enhancing the activity which was not seen with substitution to the diaryl ring with a methoxy group. ${ }^{16}$ Taken together, the SAR analysis with the carboxylic acid derivatives mentioned herein and with the carboxamide derivatives reported earlier ${ }^{16}$ indicates that a bulkier lipophilic substitution to the diaryl ring and with simple esters of the carboxylate group might provide a lipophilic-hydrophilic balance to the 4,5-diarylthiophene skeleton which led to an increase in anti-inflammatory spectrum. In conclusion, this study has identified some important substituents and its arrangement on the diaryl ring which is crucial for the anti-inflammatory property of 4,5-diarylthiophene2-carboxylic acid.

\subsection{Antioxidant activity}

The antioxidant activity of the test drug was determined using the 1,1-diphenyl-2 picrylhydrazyl (DPPH) free radical scavenging assay. The free radical scavenging activity of the synthesized compounds was assessed through their ability to quench the DPPH using ascorbic acid as a standard. The potencies for the antioxidant activity of compounds 4, 7-16 to the reference drug are shown in Table 2. In general, all the synthesized compounds were less potent than the reference compound. Among the synthesized compounds, compound 10, 11 and $\mathbf{1 3}$ exhibited slightly moderate antioxidant activity when compared to the standard.

3.4a Molecular docking studies: Molecular docking analysis was performed for the synthesised compounds with the cyclooxygenase-2 (COX-2) receptor, which is an important target for the anti-inflammatory activity. ${ }^{28}$ COX-2 is an attractive target for medicinal chemists because of its very high expression in inflamed tissues as well as many tumors. ${ }^{29,30}$ The compounds were docked using Auto Dock Tools (ADT) version 1.5.6 and Auto Dock version 4.2.5.1 docking program ${ }^{20,21}$ into the crystal structure of COX-2 receptor (PDB ID: 1PXX). ${ }^{22}$

To verify the reproducibility of the docking calculation, the co-crystallised ligand was extracted from the complex and submitted for one-ligand run calculation. Docking of co-crystallised ligand with bound X-ray conformation for 1PXX exhibits root-mean-square deviation (RMSD) value of $0.58 \AA$. This result signifies that this method is valid enough to be used for docking studies of other compounds (Figure 3A).

Docking simulation of all the synthesised compounds was performed using the same protocol of validation study. For each of the test molecules, dockings were achieved by taken into 2.5 million energy evaluations. The conformation of docked ligand with COX-2 recep- 

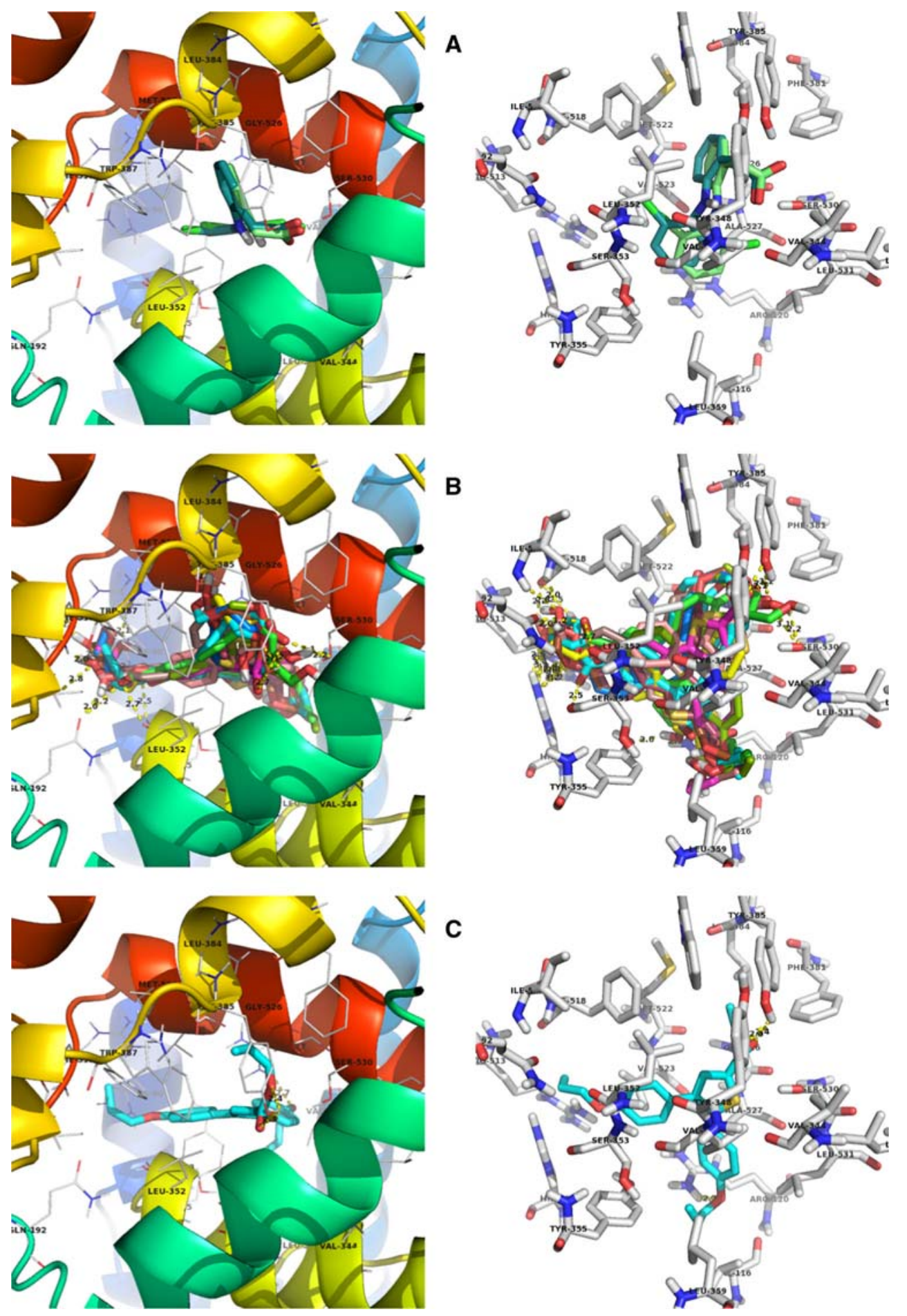

Figure 3. Molecular docking simulation with COX-2 receptor (1PXX). (A) Method validation using crystallised and docked ligand diclofenac; (B) docking simulation of all the compounds in the active site; and (C) docking simulation of the most binding energy compound 9.

tor was analysed in terms of energy, hydrogen bonding, hydrophobic and $\pi-\pi$ interaction. The final coordinates of the ligand and receptor were saved after the clear analysis of ligand-receptor interactions. Ligand and receptor interactions were investigated using
PyMol software. The free energy of binding (FEB) of all compounds were estimated and given in the Table 3.

The docking studies demonstrate that the synthesised compounds have good free energy of binding with 
Table 3. Free energy of binding (FEB) of all the synthesised compounds.

\begin{tabular}{lcc}
\hline Sl. No. & Compound & \multicolumn{2}{c}{ Free Energy of Binding $(\mathrm{kcal} / \mathrm{mol})^{\mathrm{a}}$} \\
\cline { 2 - 3 } & & COX-2 (PDB ID: 1PXX) \\
\hline 1 & $\mathbf{4}$ & -8.97 \\
2 & $\mathbf{7}$ & -9.95 \\
3 & $\mathbf{8}$ & -10.40 \\
4 & $\mathbf{9}$ & -10.48 \\
5 & $\mathbf{1 0}$ & -9.80 \\
6 & $\mathbf{1 1}$ & -9.45 \\
7 & $\mathbf{1 2}$ & -9.25 \\
8 & $\mathbf{1 3}$ & -8.90 \\
9 & $\mathbf{1 4}$ & -8.85 \\
10 & $\mathbf{1 5}$ & -8.98 \\
11 & $\mathbf{1 6}$ & -8.75 \\
12 & Diclofenac & -8.15 \\
\hline
\end{tabular}

${ }^{\mathrm{a} C a l c u l a t e d}$ using Autodock4

COX-2 receptor. Compounds display free energy of binding value from -8.75 to $-10.48 \mathrm{kcal} / \mathrm{mol}$. When compared with the standard drug diclofenac sodium, all the synthesised compounds exhibit greater binding affinity. Docking revealed that all the synthesised compounds shows various interactions such as hydrophobic, hydrophilic, $\pi-\pi$ interaction and hydrogen bonding with 25 binding site amino acids namely HIS-90, VAL-116, ARG-120, GLN-192, VAL-344, TYR-348, VAL-349, LEU-352, SER-353, TYR-355, LEU-359, PHE-381, LEU-384, TYR-385, TRP-387, ARG-513, ILE-517, PHE-518, MET-522, VAL-523, GLY-526, ALA-527, SER-530, LEU-531 and LEU-534. The docking conformation of all the compounds with COX-2 receptor is shown in Figure 3B.

Among all the compounds docked, compounds $\mathbf{8}$ and 9 exhibit very high binding with COX-2 receptor. Compound 9 shows the binding affinity of $-10.48 \mathrm{kcal} / \mathrm{mol}$ with three hydrogen bonds with two active site amino acids, namely ARG-120 and TYR-385. In Compound 9, ester carbonyl and oxygen interact with the O-H of TYR385 and forms two hydrogen bonds with the bond length of $2.3 \AA$ and $2.4 \AA$, respectively. One of the phenoxy oxygen interacts with the N-H of ARG-120 and forms a hydrogen bond with the bond length of $2.7 \AA$. In addition to the polar interactions, hydrophobic interaction was observed with the VAL-116, VAL-349, LEU-352, LEU-356, LEU-384, VAL-523, ILE-517, GLY-526 and ALA-527 amino acids. Furthermore, phenyl rings of the compound 9 display $\pi-\pi$ interaction with the phenyl rings of the TYR-355 and PHE-518. The docking conformation of compound 9 with COX-2 receptor is shown in Figure 3C.

\section{Conclusions}

In summary, a new series of bishydroxypropoxy substituted 4, 5-diarylthiophene-2-carboxylic acid derivatives were synthesized, characterized and evaluated for their in vitro anti-inflammatory activity. The in vitro anti-inflammatory activity revealed that the compound 4,5-bis(4-(3-hydroxypropoxy) phenyl)thiophene-2carboxylic acid (15) and ethyl ester (13) having antiinflammatory activity better than the standard drug diclofenac sodium. The antioxidant screening showed 4,5-bis (4-(allyloxy) phenyl) thiophene-2-carboxylic acid (10), 4,5-bis(4-(3-hydroxypropoxy)phenyl)thiophene2-carboxylic acid methyl ester (11) and 4,5-bis(4-(3hydroxypropoxy)phenyl)thiophene-2-carboxylic acid ethyl ester (13) exhibited a slightly moderate antioxidant activity than the standard ascorbic acid. Docking studies with COX-2 enzyme revealed that all the synthesised compounds exhibit greater binding affinity than the standard drug. In particular, the compounds ethyl 4,5-bis(4(allyloxy)phenyl)thiophene-2-carboxylate (8) and allyl 4,5-bis(4-(allyloxy)phenyl)thiophene-2-carboxylate (9) have high free energy binding of -10.40 and $-10.48 \mathrm{Kcal} / \mathrm{mol}$, respectively. Further studies are in progress to improve the biological activities of bishydroxypropoxy substituted 4,5-diarylthiophene-2carboxylic acid derivatives.

\section{Supplementary information (SI)}

The characterization of the compounds $\mathbf{2 - 1 6}$ using ${ }^{1} \mathrm{H}$ NMR, ${ }^{13} \mathrm{C}$ NMR, IR and Mass spectral data (Figures S1-S60) are given in the Supplementary Information, which is available at www.ias.ac.in/chemsci. 


\section{Acknowledgements}

The authors are thankful to the management of Orchid Pharma Limited, Chennai 600 119, India and Ramakrishna Mission Vivekananda College, Chennai 600 004, India for providing the required facilities.

\section{References}

1. Reitz D B and Isakson P C 1995 Cyclooxygenase-2 inhibitors Curr. Pharm. Des. 1211

2. Gadad A K, Palkar M B, Anand K, Noolvi M N, Boreddy T S and Wagwade J 2008 Synthesis and biological evaluation of 2-trifluoromethyl/sulfonamido5,6-diaryl substituted imidazo[2,1-b]-1,3,4-thiadiazoles: A novel class of cyclooxygenase-2 inhibitors Bioorg. Med. Chem. 16276

3. Sall D J, Bastian J A, Briggs S L, Buben J A, Chirgadze N Y, Clawson D K, Denney M L, Giera D D, GiffordMoore D S, Harper R W, Hauser K L, Klimkowski V J, Kohn T J, Lin H-S, McCowan J R, Palkowitz A D, Smith G F, Takeuchi K, Thrasher K J, Tinsley J M, Utterback B G, Yan S-C B and Zhang M 1997 Dibasic Benzo $[b]$ thiophene derivatives as a novel class of active site-directed thrombin inhibitors. 1. Determination of the serine protease selectivity, structure-activity relationships, and binding orientation J. Med. Chem. 403489

4. Leblanc Y, Roy P, Boyce S, Brideau C, Chan C C, Charleson S, Gordon R, Grimm E, Guay J, Léger S, Li C S, Riendeau D, Visco D, Wang Z, Webb J, Xu L J and Prasit P 1999 SAR in the alkoxy lactone series: The discovery of DFP, a potent and orally active COX-2 inhibitor Bioorg. Med. Chem. Lett. 92207

5. Lau C K, Brideau C, Chi Chung C, Charleson S, Cromlish W A, Ethier D, Gauthier J Y, Gordon R, Guay J, Kargman S, Li C-S, Prasit P, Reindeau D, Thérien M, Visco D M and Lijing X 1999 Synthesis and biological evaluation of 3-heteroaryloxy-4-phenyl-2(5H)furanones as selective COX-2 inhibitors Bioorg. Med. Chem. Lett. 93187

6. Pillarella J, Higashi A, Alexander G C and Conti R 2012 Trends in use of second-generation antipsychotics for treatment of bipolar disorder in the United States, 19982009 Psychiatr. Serv. 6383

7. Araki K, Nakanishi M and Shiroki M 1974 Thieno-(2,3e) $(1,4)$ diazepin-2-ones U.S. Patent 3849405

8. Tinney F J 1971 Tetrahydrobenzothienodiazepinone compounds U.S. Patent 3558606

9. Fink M and Irwin P 1981 Pharmacoelectroencephalographic study of brotizolam a novel hypnotic Clin. Pharmacol. Ther. 30336

10. Chan L, Das S K, Reddy T J, Poisson C, Proulx M, Pereira O, Courchesne M, Roy C, Wang W, Siddiqui A, Yannopoulos C G, Nguyen-Ba N, Labrecque D, Bethell R, Hamel M, Courtemanche-Asselin P, L'Heureux L, David M, Nicolas O, Brunette S, Bilimoria D and Bédard J 2004 Discovery of thiophene-2-carboxylic acids as potent inhibitors of HCV NS5B polymerase and HCV subgenomic RNA replication. Part 1: Sulfonamides Bioorg. Med. Chem. Lett. 14793
11. Radwan M A A, Shehab M A and El-Shenawy S M 2008 Synthesis and biological evaluation of 5 -substituted benzo[b]thiophene derivatives as antiinflammatory agents Monatsh. Chem. 140445

12. Wierzbicki M, Sauveur F, Bonnet J and Tordjman C 1998 Thiophene compounds U.S. Patent 5705525

13. Lindner M, Sippl W and Radwan A A 2010 Pharmacophore elucidation and molecular docking studies on 5-phenyl-1-(3-pyridyl)-1h-1,2,4-triazole-3carboxylic acid derivatives as COX-2 inhibitors Sci. Pharm. 78195

14. Kalgutkar A S, Crews B C, Rowlinson S W, Marnett A B, Kozak K R, Remmel R P and Marnett L J 2000 Biochemically based design of cyclooxygenase- 2 (COX-2) inhibitors: Facile conversion of nonsteroidal anti-inflammatory drugs to potent and highly selective COX-2 inhibitors Proc. Natl. Sci. U.S.A. 97925

15. Blobaum A L and Marnett L J 2007 Molecular determinants for the selective inhibition of cyclooxygenase-2 by Lumiracoxib J. Biol. Chem. 28216379

16. Shanmuganathan T, Parthasarathy K, Venugopal M, Arun Y, Dhatchanamoorthy N and Prince A A M 2017 Synthesis, in vitro anti-inflammatory activity and molecular docking studies of novel 4,5-diarylthiophene2-carboxamide derivatives J. Chem. Sci. 129117

17. Mizushima Y and Kobayashi M 1968 Interaction of antiinflammatory drugs with serum proteins, especially with some biologically active proteins J. Pharm. Pharmacol. 20169

18. Rajadurai R, Padmanabhan $\mathrm{R}$ and Ananthan $\mathrm{S}$ 2013 Synthesis and biological evaluation of diamide derivatives of (S)-BINOL and biphenyl as potential anti-inflammatory/anti-arthritic agents Med. Chem. Res. 224164

19. Shamsuzzaman, Mashrai A, Khanam H, Asif M, Ali A, Sherwani A and Owais M 2015 Green synthesis and biological evaluation of steroidal $2 \mathrm{H}$-pyrans as anticancer and antioxidant agents J. King Saud Univ. Sci. 271

20. Sanner M F 1999 Python: A programming language for software integration and development J. Mol. Graph. Model. 1757

21. Morris G M, Huey R, Lindstrom W, Sanner M F, Belew R K, Goodsell D S and Olson A J 2009 AutoDock4 and AutoDockTools4: Automated docking with selective receptor flexibility J. Comput. Chem. 302785

22. Kiefer J R, Rowlinson S W, Prusakiewicz J J, Pawlitz J L, Kozak K R, Kalgutkar A S, Stallings W C, Marnett L J and Kurumbail R G 2003 Crystal structure of Diclofenac bound to the cyclooxygenase active site of COX-2. doi:10.2210/pdb1pxx/pdb

23. Tordjman C, Sauveur F, Droual M, Briss S, Andre N, Bellot I, Deschamps C and Wierzbicki M 2003 Synthesis of the butanamide derivative $S$ 19812, a new dual inhibitor of cyclooxygenase and lipoxygenase pathways Arzneimittel-Forsch. $\mathbf{5 3} 774$

24. Wang Z, Yang Q, Bai Z, Sun J, Jiang X, Song H, Wu Y and Zhang W 2015 Synthesis and biological evaluation of 2,3-diarylthiophene analogues of combretastatin A-4 Med. Chem. Comm. 6971

25. Kvitko I Y 1969 Synthesis of derivatives of thieno[2,3$c]$ pyrazole and thieno[2,3-d]thiazoline Chem. Heterocycl. Comp. 5567 
26. Kobayashi K, Shimizu H, Sasaki A and H Suginome H 1993 Photoinduced molecular transformations. 140. New one-step general synthesis of naphtho[2,3b] furan-4,9-diones and their 2,3-dihydro derivatives by the regioselective [3+2] photoaddition of 2-hydroxy1,4-naphthoquinones with various alkynes and alkenes: application of the photoaddition to a two-step synthesis of maturinone J. Org. Chem. $\mathbf{5 8} 4614$

27. Kongkathip N, Kongkathip B, Siripong P, Sangma C, Luangkamin S, Niyomdecha M, Pattanapa S, Piyaviriyagul S and Kongsaeree P 2003 Potent antitumor activity of synthetic 1,2-naphthoquinones and 1,4-naphthoquinones Bioorg. Med. Chem. 113179
28. Arun Y, Saranraj K, Balachandran C and Perumal P T 2014 Novel spirooxindole-pyrrolidine compounds: Synthesis, anticancer and molecular docking studies Eur. J. Med. Chem. 7450

29. Bhagavat R, Saqib A and Karigar C 2012 Molecular docking studies of novel palmitoyl-ligands for cyclooxygenase-2 Chem. Bio. Drug Des. 791043

30. De Simone R, Chini M G, Bruno I, Riccio R, Mueller D, Werz O and Bifulco G 2011 Structure-based discovery of inhibitors of microsomal prostaglandin E2 synthase1,5- lipoxygenase and 5-lipoxygenase-activating protein: Promising hits for the development of new antiinflammatory agents J. Med. Chem. 541565 